\title{
Neodymium-doped glass channel waveguide laser containing an integrated distributed Bragg reflector
}

\author{
J. E. Roman and K. A. Winick \\ Electrical Engineering and Computer Science Department, University of Michigan, Ann Arbor, \\ Michigan 48109
}

(Received 26 June 1992; accepted for publication 6 October 1992)

\begin{abstract}
An integrated, distributed Bragg reflector laser in a Nd-doped, glass, channel waveguide is reported for the first time. The waveguide is fabricated using $\mathrm{Ag}^{+}$thermal ion exchange in a soda-lime-silicate-glass containing $2 \% \mathrm{Nd}_{2} \mathrm{O}_{3}$ by weight. The distributed Bragg reflector grating is produced holographically in photoresist and then etched into the waveguide using argon ion milling. The device lases in a single longitudinal mode with a pump threshold of $50 \mathrm{~mW}$ and a slope efficiency of $1 \%$.
\end{abstract}

Rare earth-doped waveguide lasers have attracted considerable attention during the last several years. These devices have been fabricated in both fiber form and as channel waveguides on planar substrates. ${ }^{1,2}$ The small active volume of the waveguide lasers results in low pump thresholds, and the devices can be made for operation in the vicinity of 1.3 and $1.5 \mu \mathrm{m}$. These wavelengths correspond to the zero dispersion and low loss spectral regions of telecommunications-grade optical fiber, respectively. In addition, waveguide lasers made in glass hosts have bandwidths on the order of tens of nanometers, and thus can support short pulse generation.

The use of a channel waveguide geometry permits the integration of multiple components on a single substrate. Integrated mode-locked ${ }^{3}$ and $Q$-switched ${ }^{4}$ lasers have in fact been demonstrated. The channel waveguide lasers fabricated, to date, have been of the Fabry-Perot type: Dielectric mirrors are either evaporated directly onto the polished waveguide ends or butt coupled to the waveguide using an index matching liquid. With evaporated mirrors, the device is mechanically stable, but the laser output is not available on the substrate. Thus, the integration of additional components is generally not possible. Furthermore, the device does not lase in a single longitudinal mode because the fluorescence bandwidth is extremely wide, and the Fabry-Perot cavity is not very frequency selective.

Narrow linewidth fiber lasers have been demonstrated using intracore Bragg gratings as distributed Bragg reflectors (DBRs)..$^{5-7}$ These gratings have been physically etched into the waveguide core. ${ }^{5}$ They have also been produced noninvasively in germanosilicate-based fibers using coherent UV radiation centered at the $244 \mathrm{~nm}$ germania oxygen-vacancy defect band. ${ }^{6,7}$

This letter reports the first (to the best of our knowledge) demonstration of an integrated distributed Bragg reflector laser in a glass channel waveguide. ${ }^{8} \mathrm{Ag}^{+}$ion exchange was used to form waveguides in a Nd-doped sodalime-silicate-glass. First-order gratings were then holographically dcfined in photoresist and subsequently etched into the waveguide using an argon ion mill to form the structure shown in Fig. 1. Lasing was achieved at an input pump power of approximately $50 \mathrm{~mW}$. The slope efficiency of the device was slightly less than $1 \%$, and lasing occurred in a single longitudinal mode. Fabrication and characterization of the laser is described below.

A soda-lime-silicate-glass containing by weight percent $71.2 \% \mathrm{SiO}_{2}, 2.01 \% \quad \mathrm{Nd}_{2} \mathrm{O}_{3}, 16.2 \% \quad \mathrm{Na}_{2} \mathrm{O}, 1.10 \% \mathrm{~K}_{2} \mathrm{O}$, $4.92 \% \mathrm{CaO}, 3.49 \% \mathrm{MgO}, 0.903 \% \mathrm{Al}_{2} \mathrm{O}_{3}, 0.0502 \% \mathrm{Sb}_{2} \mathrm{O}_{3}$ was cut into pieces approximately $1 \mathrm{in}$. on a side and $1-\mathrm{mm}$ thick. Waveguides were then formed by performing $\mathrm{Ag}^{+}$ thermal ion exchange (0.2 wt \% $\mathrm{AgNO}_{3}$ and $99.8 \mathrm{wt} \%$ $\mathrm{NaNO}_{3}$ ) through a $4-\mu \mathrm{m}$-wide diffusion mask at $340{ }^{\circ} \mathrm{C}$ for $8 \mathrm{~min}$. Grating fabrication proceeded next. The top surface of the substrate was coated with photoresist and holographically exposed using a $\mathrm{HeCd}$ laser operating at 442 nm. During development, the grating diffraction efficiency was monitored, and development was stopped at the point of maximum diffraction efficiency. ${ }^{9}$ The developed photoresist grating pattern was subsequently etched into the waveguide to a depth of $600 \AA$ using an argon ion mill. The etch time was approximately $4 \mathrm{~min}$. The resulting grating was $5.5-\mu \mathrm{m}$ long with a period of $0.35 \mu \mathrm{m}$.

The waveguides were characterized before grating fabrication. First, $\mathrm{Ag}^{+}$ion exchanged planar waveguides were made in order to estimate the surface index change and guide depth. Prism coupling was employed to determine the modes of the planar guide. The surface index change and $e^{-1}$ waveguide depth were 0.046 and $1.3 \mu \mathrm{m}$, respectively. These values were computed by assuming a complementary error function refractive index profile $\mathrm{e}^{\mathrm{l0}}$ and using the inverse WKB technique. ${ }^{11}$ The loss of the channel waveguides, measured by the cut-back technique, ranged

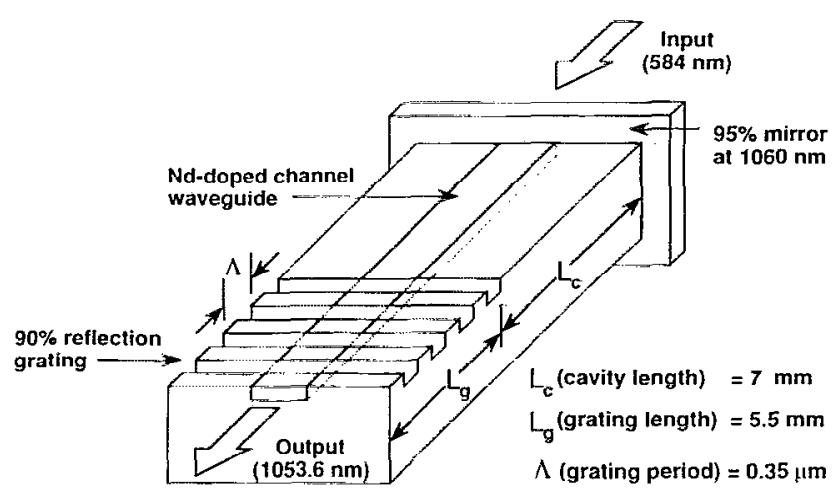

FIG. 1. Distributed Bragg reflector laser. 


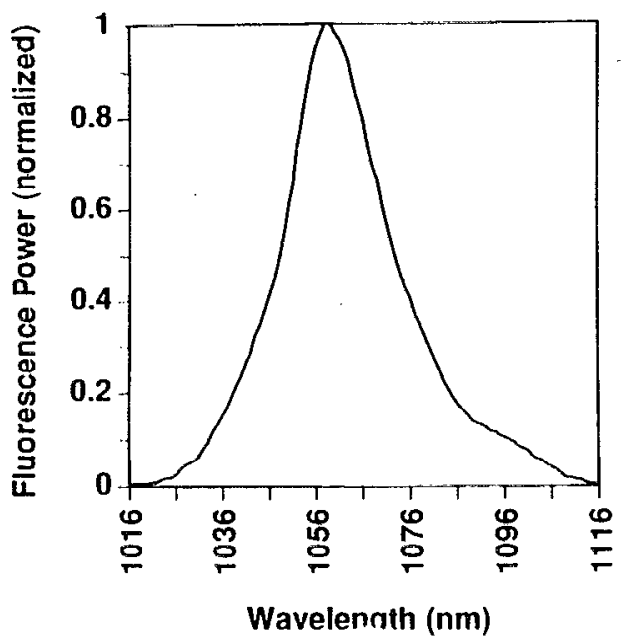

FIG. 2. Fluorescence spectrum of Nd-doped glass.

between 1 and $1.8 \mathrm{~dB} / \mathrm{cm}$. Channel waveguides made in the same glass using $\mathrm{K}^{+}$ion exchange had losses consistently in the neighborhood of $1 \mathrm{~dB} / \mathrm{cm}$. These waveguides were considerably deeper and had a smaller surface index change than those made with $\mathrm{Ag}^{+}$ion exchange. The surface quality of the substrates were not particularly good, and thus shallower waveguides would be expected to have higher loss. The fluorescence spectrum of the glass was measured using a $1 / 4 \mathrm{~m}$ monochromator and is shown in Fig. 2. The $e^{-1}$ fluorescence lifetime from the ${ }^{4} \mathrm{~F}_{3 / 2}$ metastable level was found to be $360 \mu \mathrm{s}$, and the glass absorption coefficient had a value of $3.5 \mathrm{~cm}^{-1}$ at the pump wavelength of $584 \mathrm{~nm}$.

As a first step, 95\% reflecting dielectric mirrors at 1.06 $\mu \mathrm{m}$ were coated on microscope slides and butted against the end of the waveguides using a fluorinated index matching liquid. A 13-cm-long waveguide (without a grating)

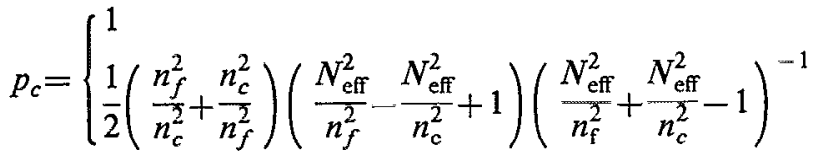

where $L_{g}$ is the grating length, $h_{\text {eff }}$ is the effective waveguide depth, $N_{\text {eff }}$ is the effective index of the waveguide mode, and $n_{f}, n_{c}$, and $n_{s}$, are the refractive indices of the waveguide layer, cover layer, and substrate layer, respectively. The full-width-null-to-null bandwidth, $\Delta \lambda$, of the grating filter is ${ }^{12}$

$$
\Delta \lambda=\frac{2 \Lambda \lambda}{L_{g}}\left[1+\left(\kappa L_{g} / \pi\right)^{2}\right]^{1 / 2},
$$

where $\Lambda$ is the grating period. As a conservativc cstimate, we assumed, $h_{\text {eff }} \approx 2 \mu \mathrm{m}, n_{f}-N_{\text {eff }} \approx 0.01, n_{f} \approx n_{s} \approx 1.5$, and $n_{c}=1$. Thus, $R_{\max }=84 \%$ (TE mode), $R_{\max }=5.3 \%$ (TM mode), and $\Delta \lambda=1.4 \AA$ (TE mode). The fluorescence from made with a $4-\mu \mathrm{m}$-wide diffusion mask, was then end-fire pumped from a dye laser at $584 \mathrm{~nm}$. The output spectrum was measured using a $1 / 4 \mathrm{~m}$ monochromator and was nominally 5 -nm wide. The device had a pump threshold and slope efficiency of approximately $50 \mathrm{~mW}$ and $0.5 \%$, respectively.

A $5.5-\mathrm{mm}$-long square wave grating was etched into the waveguide as described above. The grating period, $\Lambda$, was chosen to yield maximum reflectivity in the vicinity of $1.06 \mu \mathrm{m}$. It follows from the Bragg condition

$$
\Lambda=\frac{\lambda_{0}}{2 N_{\text {eff }}},
$$

where $\lambda_{0}$ is the wavelength at peak reflectivity, and $N_{\text {eff }}$ is the effective index of the guided mode. Since $N_{\text {eff }} \approx n_{\text {glass }}$ $\approx 1.5, \Lambda \approx 0.35 \mu \mathrm{m}$. The grating depth was calculated by illuminating the grating from the top using a $\mathrm{HeCd}$ laser $(442 \mathrm{~nm})$ and measuring the diffraction efficiency, $\eta$, into the first order. The diffraction efficiency is given by

$$
\eta=\frac{2}{\pi^{2}}\left\{1-\cos \left(\frac{2 \pi}{\lambda}\left(n_{s}-1\right) d\right)\right\},
$$

where $\lambda$ is the wavelength of the illuminating light, $n_{s}$ is the substrate refractive index, and $d$ is the peak-to-peak grating depth. $\eta$ was measured to be $1.7 \%$, which corresponds to a grating depth of approximately $600 \AA$. This value was verified by an electron microscope picture of the grating. The peak reflectivity, $R_{\max }$, and bandwidth, $\Delta \lambda$, of the grating were estimated assuming that the waveguide was planar and that the refractive index profile was a step. For such a structure $^{12}$

$$
\begin{aligned}
& R_{\max }=\tanh ^{2}\left(\kappa L_{g}\right), \\
& \kappa=\frac{1}{\lambda} \frac{d}{h_{\mathrm{eff}}} \frac{\left(n_{f}^{2}-N_{\mathrm{eff}}^{2}\right)}{2 N_{\mathrm{eff}}} p_{c},
\end{aligned}
$$

for TE mode

for TM mode

a $\mathrm{Nd}$-doped fiber was coupled into the waveguide, and a $1 / 4 \mathrm{~m}$ monochromator, having a full-width-half-maximum resolution of $3 \AA$, was used to measure the spectral transmittance of the grating. The maximum reflectivity occurred at a wavelength of $1052 \mathrm{~nm}$, and the grating bandwidth was less than the $3 \AA$ resolution of the monochromator.

A $95 \%$ reflecting dielectric mirror was butt coupled, using a fluorinated index matching liquid, to the waveguide end opposite the grating. The waveguide length between the grating and the mirror was $7 \mathrm{~mm}$, and thus the longitudinal mode spacing was approximately $14 \mathrm{GHz}(0.52$ $\AA$ ). Using a $20 \times$ microscope objective as a coupling lens, 


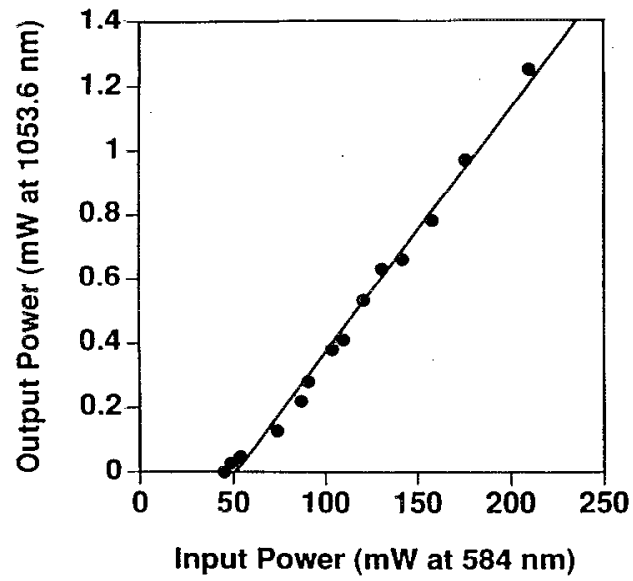

FIG. 3. Lasing characteristics of DBR waveguide laser.

the device was end-fire pumped through the mirror by a dye laser operating at $584 \mathrm{~nm}$. It was not possible to endfire pump the waveguide at the opposite end, since the pump coupled to radiation modes via the grating. Lasing was achieved at a pump power threshold of $50 \mathrm{~mW}$ and the measured slope efficiency was $1 \%$, as indicated in Fig. 3. The output spectrum was measured using a scanning Fabry-Perot spectrometer having a free spectral range of $7.5 \mathrm{GHz}$ and a nominal bandwidth of $37.5 \mathrm{MHz}$. As indicated in Fig. 4, the laser operated in a single longitudinal mode with a linewidth (short term) below the $37.5 \mathrm{MHz}$ spectrometer resolution. Instabilities, which we believe are

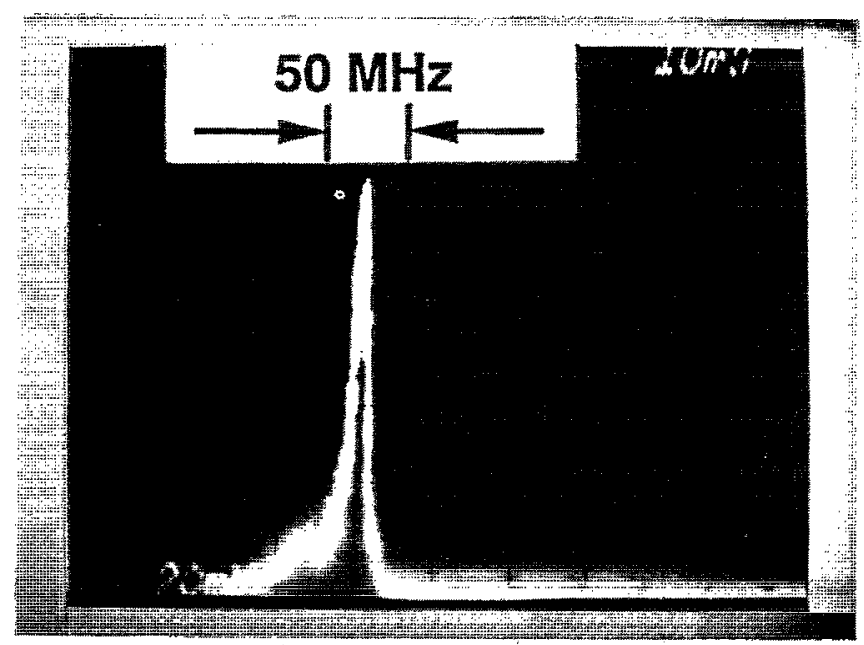

FIG. 4. DBR laser linewidth measurement. due to the butt-coupled mirror at one end of the cavity, cause the center frequency to slowly jitter by $\pm 250 \mathrm{MHz}$.

We note that ion-exchangeable glass can be easily produced with the high rare earth-doping levels needed to absorb the pump in a short distance. Thus, the cavity length of a channel waveguide laser is generally shorter than that of a fiber laser device. This short cavity length increases the longitudinal mode spacing and makes single longitudinal mode operation more likely to occur.

As expected, the laser output was polarized TE, because the reflectivity of the distributed Bragg grating was much lower for the TM mode. The output laser powers, $P_{1,2}$, emitted from each end of the device (1 is grating end, 2 is mirror end) were measured. The ratio of these powers was then used to compute the grating reflectivity, $R_{1}$, at the lasing wavelength by using the relationship

$$
\frac{P_{1}}{P_{2}}=\frac{1-R_{1}}{1-R_{2}} \sqrt{\frac{R_{2}}{R_{1}}},
$$

where $R_{2}$, the mirror reflectivity, equals 0.95 . The measured ratio, $P_{1} / P_{2}$, together with Eq. (7) gave a grating reflectivity value of $90 \%$.

In conclusion, the first demonstration of an integrated, distributed Bragg reflector, rare earth-doped, glass, channel waveguide laser is reported. The device operates in a single longitudinal mode, with an output linewidth less than $37.5 \mathrm{MHz}$. Optimization of the glass, waveguide structure, and grating parameters are expected to improve device performance significantly.

This work was supported by the National Science Foundation under Grant No. ECS 890-8902.

'Y. Hibino et al., IEEE Photon. Technol. Lett. 1, 349 (1989).

${ }^{2}$ N. A. Sanford, K. J. Malone, and D. R. Larson, Opt. Lett. 15, 336 (1990).

${ }^{3}$ E. Lallier, J. -P. Pocholle, M. Papuchon, Q. He, M. De Micheli, D. B. Ostrowsky, G. Grezes-Besset, and E. Pelletier, Electron. Lett. 27, 936 (1991).

${ }^{4}$ E. K. Mwarani, D. M. Murphy, M. Hempstead, L. Reekie, and J. S. Wilkinson, Photonics Technol. Lett. 4, 235 (1992).

${ }^{5}$ I. M. Juancey, L. Reekie, J. F. Townsend, D. N. Payne, and C. J. Rowe, Electron. Lett. 24, 24 (1988)

${ }^{6}$ G. A. Ball, W. W. Morey, and J. P. Waters, Electron. Lett. 26, 1829 (1990).

${ }^{7}$ G. A. Ball, W. W. Morey, and W. H. Glenn, Photon. Technol. Lett. 3, 613 (1991).

${ }^{8}$ K. A. Winick and J. E. Roman, Annual Optical Society of America Meeting, Albuquerque, NM, Sept. 1992 (unpublished), Postdeadline Paper PD9.

${ }^{9} \mathrm{~L}$. $\mathrm{Li}, \mathrm{M} . \mathrm{Xu}, \mathrm{G}$. Stegeman, and C. T. Seaton, Proc. SPIE 835, 72 (1987).

${ }^{10}$ R. K. Lagu and R. V. Ramaswamy, J. Lightwave Technol. LT-4, 176 (1986).

${ }^{11}$ J. E. Gortych and D. G. Hall, Quantum Electron. QE-22, 892 (1986).

${ }^{12}$ Guided-Wave Optoelectronics, edited by T. Tamir (Springer, New York, 1988), Chap. 2 